\title{
Time Slotted Channel Hopping for Smart Metering: Measurements and Analysis of Medium Access
}

\author{
Mikhail Vilgelm, Murat Gürsu, Samuele Zoppi, Wolfgang Kellerer \\ Chair of Communication Networks \\ Technical University of Munich, Germany \\ Email:\{mikhail.vilgelm, murat.guersu, samuele.zoppi, wolfgang.kellerer\}@tum.de
}

\begin{abstract}
The paper presents an indoor testbed evaluation of a multi-hop IEEE 802.15.4e Time Slotted Channel Hopping (TSCH) sensor network, operating in $2.4 \mathrm{GHz}$ band. First goal of the evaluation is to assess the perspectives of TSCH, as a more reliable version of IEEE 802.15 .4 , for meter reading applications in a Smart Grid. Secondly, we compare the network performance for two medium access schemes: contention-based and contention-free. We model two automatic meter reading applications, representing regular metering readings and alarm reporting. With this model, we have collected eight measurement data sets for different interference and load conditions. Based on the data sets, link- and end-to-end reliability, and end-to-end delay are analyzed. Our results point out that the delay constraints can be satisfied. However, channel hopping is insufficient for meeting the reliability requirements, and additional mechanisms to couple with high link-layer losses are required.
\end{abstract}

\section{INTRODUCTION}

Smart grid (SG) is a concept of a new generation power grid, with disruptive enhancements in power production, operation and consumption [1], [2]. SG energy production is aided with distributed renewable energy, allowing to utilize more efficient and environment friendly power sources. The operation of SG is envisioned to be reliable and resistant to failures, due to high penetration of monitoring devices and the distributed nature of the production. Finally, energy consumption in SG is more intelligent and flexible compared to the legacy grid. It relies on such concepts as dynamic pricing, where the distribution system operator can change the price of the power on the daily or hourly basis, thus, steering the consumption towards less busy hours, and avoiding the peak loads. The key component behind the SG is the communication system, coupling together consumer, producer and operator of the grid.

The part of the SG, which we selected for the consideration in this paper, are the Automatic Meter Readings (AMR) [1]. It can include several applications, such as conventional meter readings or reporting of meter and grid events and alarms. For AMR deployment, there have been many communication architectures proposed, including power-line communication, wired connections, and several wireless [3], [4]. Many of the proposed architectures assume the usage of Wireless Sensor Network (WSN), either standalone [5], or as part of a two-tier model, in a combination with cellular networks [6]. WSN have such advantages as ease of deployment, low hardware costs, and highly customizable firmware.

However, current wireless Machine-to-Machine (M2M) applications, and SG among them, are characterized by increas- ing demands in latency and reliability. This forces the focus shift in wireless standards' development from increasing the maximum data rate to investigating means of delivering the data deterministically on time and with minimum losses. The new focus has been adopted by $5 \mathrm{G}$ wireless technology vision in a concept of ultra-low-latency and ultra-high-reliability communication [7]. On the other hand, WSN are inherently unreliable, since they operate in the unlicensed frequency spectrum, and interfere with other wireless technologies [8]. To address this issue, IEEE 802.15.4 standard for WSN has been recently updated with amendment " $e$ " [9], defining the time division multiple access (TDMA) based medium access contol (MAC) for deterministic behavior as well as frequency hopping for decreasing the interference effects.

Recent work aims at evaluating performance aspects of 802.15.4e, however, mostly focusing on simulations of single hop scenarios. Among others, simulative assessments of delay and packet loss in a single hop scenario have been done in [9], [10]. The authors in [9] show that 802.15.4e can keep the worst case packet delay bounded until a certain load in the network is induced. In [10], two different MAC profiles are compared, with the outcome that time slotted channel hopping (TSCH) is preferable in terms of end-to-end delay for small networks, while deterministic and synchronous multi-channel extension (DSME) is more suitable for large networks ( $>30$ nodes).

There is however less work on testbed deployments of the 802.15.4e WSN. In [11], [12], reliability aspects of TSCH have been examined in a single hop scenario. In contrast to them, we consider a multi hop network, and study both delay and reliability. Although Gonga et. al [13] do not address 802.15.4e directly, it is important to mention it as comprehensive testbed evaluation of WSN. They study frequency hopping and compare its reliability benefits to the adaptive routing solution. Our paper primarily focuses on the delay aspect and compares two different frame configuration scenarios, thus, studying the effects of time slot allocation.

In this paper, we present an indoor testbed evaluation of a multi-hop 802.15.4e WSN deployment in $2.4 \mathrm{GHz}$ band. The goal of the evaluation is twofold. Firstly, we aim at assessing the suitability of TSCH as a MAC protocol for AMR. For that, we use an AMR data aggregation setup in an industrial facility or in a campus, with application models of (1) regular metering readings and (2) meter alarm reporting. The second, more general, goal is to evaluate the performance of $\mathrm{TSCH}$ 
with two different MAC operating modes: reserved and shared slots, both using frequency hopping technique. For that, we measure link- and network-level reliability, and end-to-end delay. We consider three different interference scenarios and two network load conditions.

The paper is structured as follows. Sec. II introduces the background on protocol stack. Measurement scenario and setup are introduced in Sec. III. Sec. IV describes the observations about the data set and its parameters, and Sec. V illustrates the network operation, reliability and delay measurements. We discuss the suitability of TSCH for AMR in Sec. VI and, finally, conclude with Sec. VII.

\section{BACKGROUND}

In this section, we introduce essential background on the protocol stack. It consists of the IEEE 802.15.4 physical layer, 802.15.4e TSCH as medium access layer, and IPv6/6loWPAN as network/adaptation layer. Routing in a network is established via IETF Routing over Low Power Lossy Networks (RPL) [14]. In the following, we are outlining MAC, network, and application layers.

\section{A. MAC layer}

IEEE $802.15 .4 \mathrm{e}$ has two main differences to its predecessor 802.15.4. Firstly, it adds time division multiple access to allow determinism in the end-to-end delay: time slots can be reserved for certain nodes in network, hence, avoiding collisions and theoretically improving the reliability. As a second feature, frequency diversity techniques, such as channel hopping, have been introduced to coupe with the inter-technology interference in the $2.4 \mathrm{GHz}$ unlicensed band. The standard defines three profiles, suitable for different applications. Here, we use TSCH profile, as it provided better delay for the small networks [10]. The superframe structure is depicted in Fig. 11. A superframe is comprised of the two kinds of slots: ADV, reserved for advertising the schedule and absolute slot number (ASN) with beacons, and TX/RX slots for transmitting regular data. The standard does not specify how the slots should be allocated to different nodes. Here, we employ two variations: contention-free with (1) reserved slots, and contention-based with (2) shared slots. Frequency hopping is implemented in the following way. Nodes in a network choose the frequency in a coordinated way, based on the ASN. 802.15.4 channel at the $i$-th slot is chosen from a hopping sequence $\mathrm{HS}$ as: $c_{i}=\operatorname{HS}\left[\operatorname{ASN}_{i} \% 16\right]$.

\section{B. Network layer}

For addressing and adaptation we use IPv6/6loWPAN, while the routing is accomplished using RPL. This protocol has been designed with the primary use in low power and lossy network (LLN), and with the application data being sent in a "one-to-many" (broadcast) or a "many-to-one" (convergecast) fashion. Hence, the protocol reduces the network topology to a destination-oriented directed acyclic graph (DAG), where every node is assigned a DAG rank according to its position relative to the destination (aka DAG root. Based on the DAG,

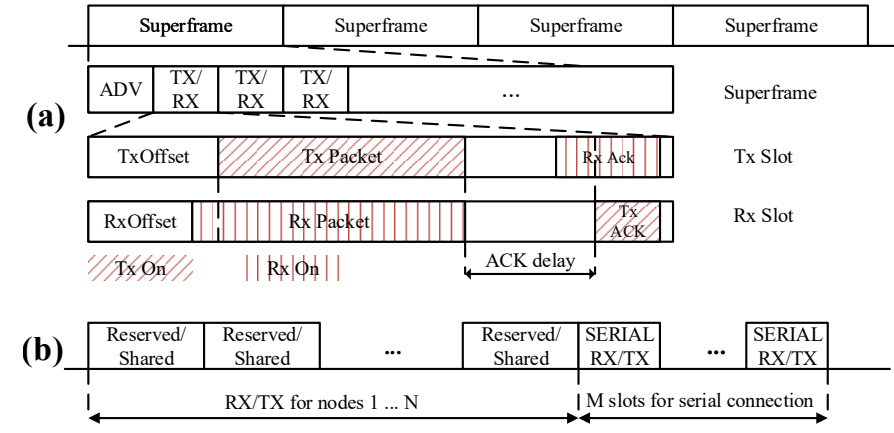

Fig. 1. Superframe structure of 802.15.4e TSCH: (a) generalized, (b) our setup - one superframe: frame length 17 slots $\times 15 \mathrm{~ms}=255 \mathrm{~ms}$. In (b), a superframe additionally contains $\mathrm{M}$ slots for delivering data from the nodes to a PC via serial interface.

network routes are computed in way that optimizes broadcast and convergecast in such network. RPL support link or node properties as cost metrics. The routing tree is constructed hopby-hop, where every node choses the parent node based on the DAG rank (see example in Fig. 3). The link cost metric is expected transmission count (ETX) [15].

\section{AMR application model}

In order to propagate the characteristics of AMR on to communications, we model two application modes as in [16]: regular periodic and bursty application. The periodic applications creates a dummy message of size $L_{\mathrm{p}}$ and sends it to sink with period $T_{\mathrm{P}}$. The bursty application aims to emulate periods of high load in the network, e.g., caused by the meters' or grid alarm reporting in a case of outage. This application has a sleeping phase and the burst phase with durations $T_{\mathrm{S}}$ and $T_{\mathrm{B}}$ respectively. In the burst phase, the node is sending frequent packets with a short inter-packet interval $T_{\mathrm{BP}}$. The idea behind it is to observe how the network behaves in a situations of sudden overloads. The lengths of all periods are selected according to the network load. We restrict our evaluation to up-link directed traffic, thus, we do not model dynamic pricing and other down-link intensive AMR applications.

\section{SETUP}

Our setup comprises a total of 13 nodes randomly distributed in a realistic working environment composed by offices and shared facilities. Every node is a Zolertia Z1 device, and runs the same stock firmware OpenWSN from Watteyne et. al [17] implementing the 802.15.4e MAC access scheme. The firmware has been modified in order to implement a schedule that enables designated time slots 1 The data was collected for two MAC variations and for three background interference scenarios.

Applications. Among the 13 nodes, we distinguish different groups according to the type of application layer that generates the messages. Thus, we have the following groups of nodes with relative range of addresses: node 1 (sink, DAG root), nodes 2-8 with periodic application, nodes 9-11 with bursty

\footnotetext{
${ }^{1}$ Project's source code has been altered according to our setup. The repositories with our versions are available online (release LKN_v2.0): https:// github.com/tum-lkn/openwsn-fw and https://github.com/tum-lkn/openwsn-sw
} 
TABLE I

TRAFFIC PARAMETERS.

\begin{tabular}{|c||c||c||c||c||c||c|}
\hline \multicolumn{1}{|c||}{} & \multicolumn{2}{c||}{ Periodic } & \multicolumn{4}{c|}{ Bursty } \\
\hline & $L_{\mathrm{p}}$ & $T_{\mathrm{P}}$ & $L_{\mathrm{p}}$ & $T_{\mathrm{S}}$ & $T_{\mathrm{B}}$ & $T_{\mathrm{BP}}$ \\
\hline \hline High load & 80bytes & $2 \mathrm{~s}$ & 80 bytes & $10 \mathrm{~s}$ & $3 \mathrm{~s}$ & $0.5 \mathrm{~s}$ \\
\hline Low load & 80bytes & $5 \mathrm{~s}$ & 80 bytes & $10 \mathrm{~s}$ & $3 \mathrm{~s}$ & $0.7 \mathrm{~s}$ \\
\hline
\end{tabular}

application. Also, nodes 12 and 13 do not have own application and only serve as forwarders. We conduct the measurements for two load scenarios: high and low overall load. Traffic parameters are summarized in Table [

Medium access. As mentioned before, the tests were performed for two different TSCH variations: with shared (data sets V-VIII) and reserved slots (data sets I-IV). The superframe structure is the same in both cases (see Fig. 1b). $N=13$ slots are used for transmitting the data, and $M=4$ for forwarding the data to the computer. In the reserved case, slots 1-13 are assigned to one of the node respectively. In the shared case, the nodes may send in any slot, thus, the collisions might occur. To resolve a collision, exponential back-off from 2 to 3 (expressed in slot duration $t_{\mathrm{s}}=15 \mathrm{~ms}$ ). We further limit the transmission attempts for a given packet to $W^{\max }=3$. The nodes use the default hopping sequence with 16 channels [17].

Interference scenarios. The measurements are conducted under three interference scenarios. First two scenarios correspond to weekend (data sets I and V) and week days (II and VI), respectively. Since the office environment has several wireless local area network (WLAN) access points (AP) and clients, it is expected that the weekends and week days might have different interference levels. Additionally, data sets III, IV and VII, VIII were collected with artificially induced high interference level, with two access points on WLAN channels 1 and 5, and three users connected to them. The users' duty cycle has been split into alternating sleeping and active phases with 100 s durations. During the active phase, users fully utilize the available bandwidth with UDP applications (created by iperf).

Deployment. The nodes were deployed unsynchronized in the positions depicted in the map of Fig. 3. Then, the node with address 1 is connected to the PC via serial port and is programmed as DAG root, enabling synchronization of the network through periodic beaconing. In all the measurements, the DAG root is used as a sink, it receives all the messages and forwards them to the PC for data analysis. In order to collect and analyse data from our scenario, for every packet generated at the application layer of every device, we collect the following information (see Fig. 2): source information, ASN of reception time, application sequence number, and per hop information (consisting of transmitter address, transmission frequency, received signal strength indicator (RSSI), and the retransmission counter: number of times that the transmission of the packet was attempted).

\section{DATA SETS}

Three different interference scenarios and two different MAC schemes resulted in six data sets collected. For both
Hop 1

\begin{tabular}{|l|l|l|l|l|l|l|}
\hline Src ASN & App SN & Addr & TX Freq & RSSI & ReTX cnt & $\cdots$ \\
\hline - Source info & \multicolumn{5}{|c|}{ Hop 1...N info } \\
\hline
\end{tabular}

Fig. 2. Visual representation of data collected for every packet.

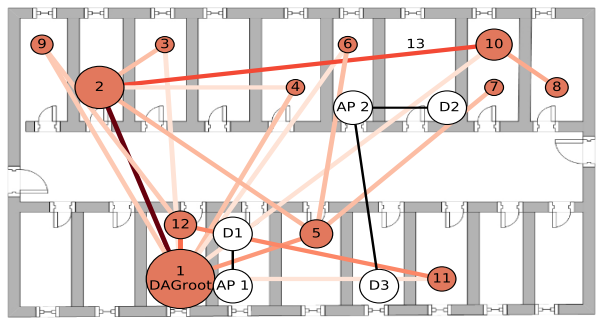

Fig. 3. Exemplary topology and routing paths in the working environment (data set VII). Color gradient represents the relative usage of the link.

MAC schemes we performed measurements for induced high interference and high load scenarios, hence, in total, eight data sets have been obtained ${ }^{2}$ All the parameters of the data sets are summarized in Table III It is shown that every scenario contains similar numbers of total and average generated packets per node, and over different frequency channels. Exceptions to it are the high load measurements: they were performed in a shorter time, hence, less packets generated.

Among different scenarios, the data sets experience differences due to the fluctuating behavior of the wireless channel and, hence, different resulting RPL paths. To exemplify the routes in the network, Fig. 3 presents all the used links between the nodes for data set VII (induced interference). The figure shows the positions of the nodes in the environment. The links between the nodes represent packet transmissions: their color gradient shows relative total number of transmitted packets over that link. The effect of the interference on the link choice is observed. The links 10-5, 11-12, 5-1 (more affected by interferers) are less popular and are partially substituted by the links 10-2, 5-2 for this scenario interference scenario.

\section{Performance Evaluation}

This section presents the network operation effects we observed, and reliability and delay analysis.

\section{A. Network Effects}

Two important network operation effects are observed for all data sets: delivery of duplicated packets (Table III) and outof-order delivery (OOD) (Fig. 4). The first effect is explained by the fact that MAC resends the packet in the case of a lost acknowledgement, and the receiver does not filter the packet which are already forwarded. The duplication has affected all data sets, and, on average, both applications equally strong. This effect can lead to creating unnecessary load in the network, and, can be mitigated if the nodes will keep track on the already received and forwarded packets.

\footnotetext{
${ }^{2}$ The data gathered during the experiments and the processing tools are available here: https://github.com/tum-lkn/tschdata
} 
TABLE II

SUMMARY OF THE DATA SETS PARAMETERS.

\begin{tabular}{|c||c||c||c||c||c||c||c||c|}
\hline \multicolumn{1}{|c||}{} & \multicolumn{4}{c||}{ Reserved slots } & \multicolumn{4}{c|}{ Shared slots } \\
\hline & Weekend & Week day & High IF & High Load, High IF & Weekend & Week day & Induced IF & High load, High IF \\
\hline Data set & I & II & III & IV & V & VI & VII & VIII \\
\hline \hline Duration (min) & 211 & 243 & 207 & 44 & 203 & 230 & 188 & 93 \\
\hline Packet gen. per node & 8175 & 7689 & 6387 & 1550 & 7626 & 8591 & 7516 & \\
\hline Packet gen. per channel & 4078 & 3664 & 3262 & 773 & 4073 & 4096 & 3705 & 2710 \\
\hline Packet gen. total & 32862 & 33644 & 27579 & 6481 & 33977 & 37546 & 30899 & 21611 \\
\hline Duplicated packets & 0.1174 & 0.0905 & 0.3450 & 0.2476 & 0.1269 & 0.0689 & 0.1526 & 0.1994 \\
\hline
\end{tabular}
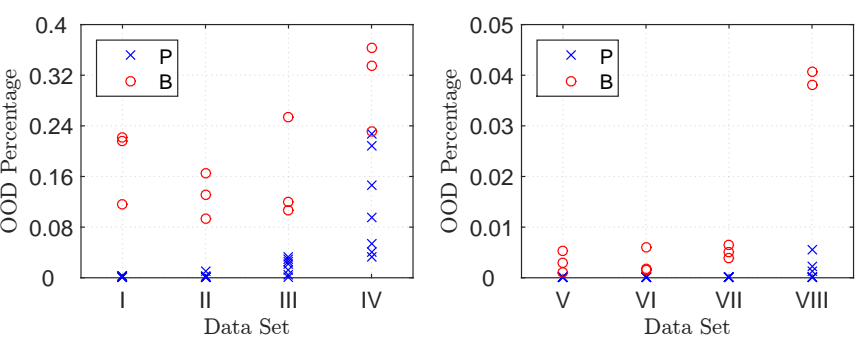

Fig. 4. OOD distribution for periodic (labeled as P) and bursty (B) application. Left plot shows dedicated slots scenario, while right - shared slots (see Table III). Type-P OOD is calculated according to RFC4737.

Second effect, out-of-order delivery, is observed primarily in the dedicated slots medium access (data sets I-IV) and in the high-load shared slots scenario (VIII). It is partly caused by the buffer implementation and by the dynamic topology changes by RPL: some of the packets are forwarded and when the route has changed the next packet may arrive faster. Further investigation points out that the bursty applications result in higher OOD than periodic one. This result suggest that high application layer periodicity can cause problems on OOD. It also suggests, that if the metering application is sensitive to the delivery order, a transport layer protocol should be deployed for reordering (e.g., UDP+RTP).

\section{B. Reliability}

We define end-to-end reliability as the ratio of packets successfully received on a DAG root (Packet Delivery Ratio, PDR). A packet is dropped if its reception has been unsuccessful for $W^{\text {max }}$ transmissions, or if the buffer is full. We exclude the effect of the buffer by setting the mote to produce an error

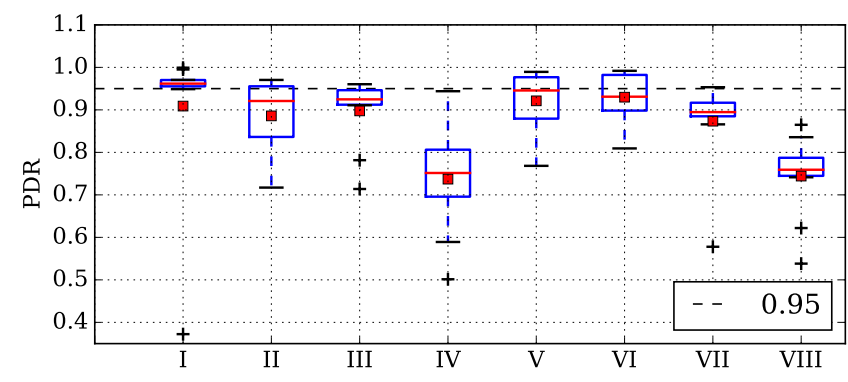

Fig. 5. Packet Drop Rate (PDR) distribution for different scenarios (numeration according to Table $\Pi$. Mean values are represented with red squares. Left four scenarios are for dedicated slots, right four - shared slots. if the overflow occurs, thus, we observe only the MAC effects against channel state.

First, we observe the effects of interference on the per channel link loss ratio from Fig. 6 For dedicated slots, link loss ratio is calculated from packets received with the certain number of transmission attempts. If a packet is received after two retransmissions on the same hop, we can deduce the channels on which the packets were lost via the HS. However, this is not possible with shared slots scheme due to the random back-off timer. In order to compensate for this, we collect the number of re-transmissions as packet losses before the successful reception. Then we distributed these on all of the channels since the probability of selecting a channel is uniformly random. On top of these failure, we take additionally into account the success of the channels over which we received a packet, and calculated the packet transmission rate accordingly. The effects of collisions are observed from the difference between the shared and the reserved case in Fig. 6 The difference between week days and weekends is not clearly distinguishable, while the induced interference effect is more visible. WLAN channel 1 (intercepting with WSN channels 11 - 14) is dominant, whereas the effect of interference of WLAN channel 5 (WSN channels $15-18$ ) is only observable in certain nodes such as 6,10 , and 12 . This is related to the placement of the nodes (Sec. III): WLAN channel 1 is interfering with a larger amount of links.

Collisions were expected to be a problem for PDR of the shared slots case. Both MAC schemes use channel hopping, hence, they are similarly resistant to cross-technology interference. However, the measurement results in Fig. 5 show that the there is no significant difference between shared and dedicated slots: PDR is higher for shared slots in the weekend and week day measurements (I,II vs V,VI), but lower in the high interference measurements (III vs VII). The deviation is however below $5 \%$. This suggests that the collision resolution works well for this load scenarios. It is also observed that high load, high interference scenarios IV and VIII record significantly lower PDR.

\section{Delay}

Throughout this section, we use several metrics to analyze the delay. First, $d_{\mathrm{p}}$ is the end-to-end delay for a single packet, as measured. In order to mitigate the routing effect, while comparing different applications, we also use per packet hop delay $d_{\text {hop }}=d_{\mathrm{p}} / n$, where $n$ number of hops. 


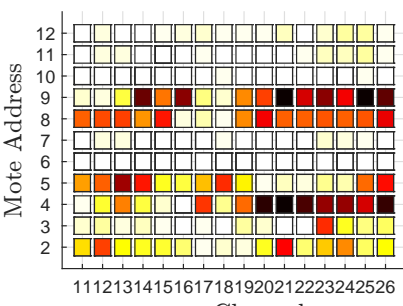

Channels

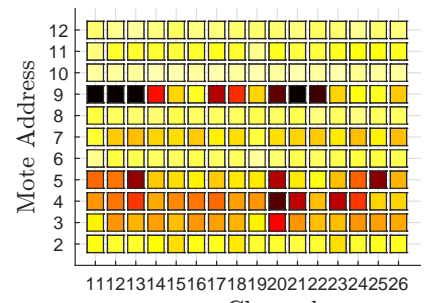

Channels

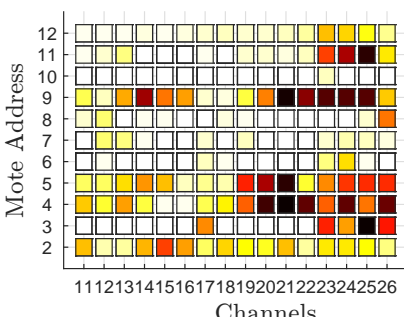

Channels

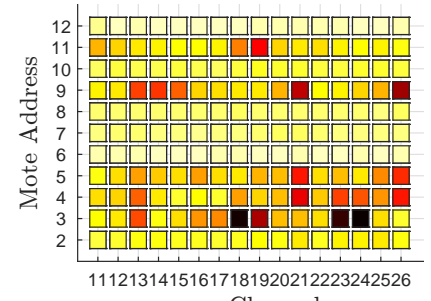

Channels

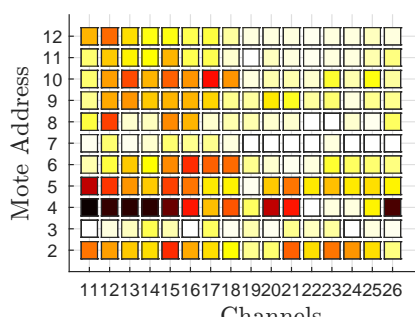

Channels

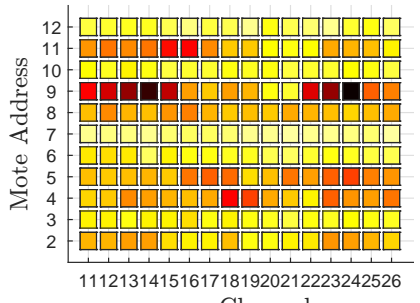

Channels

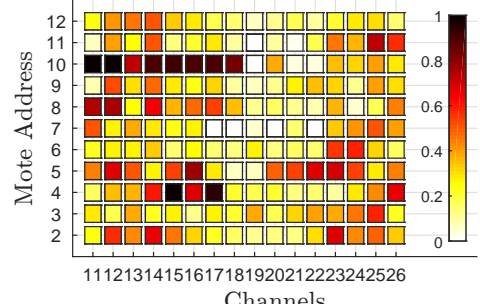

Channels

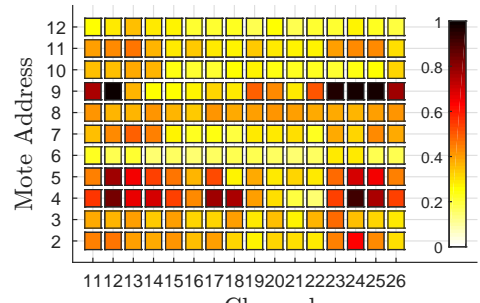

Channels

Fig. 6. Channel based packet loss rates, selecting the best neighbor for each node. From left to right: weekend (I,V), week day (II, VI), high interference (III, VII), and high load (IV, VIII) scenarios. Dedicated and shared slots on upper and lower part respectively.

Furthermore, to better differentiate sources of the delay, we introduce the following additional metrics: minimum path delay $d_{\min }$, which is the minimum achievable delay on given path; and minimum path delay with retransmissions $d_{\text {retx }}$, which is the minimum achievable delay on a path, given the transmission attempts for every hop on the path:

$$
\begin{aligned}
d_{\min } & =\frac{t_{\text {frame }}}{2}+t_{\mathrm{s}-\mathrm{d}}(n-1) \\
d_{\mathrm{retx}} & =\frac{t_{\text {frame }}}{2}+\sum_{i=2}^{n}\left(t_{\mathrm{s}-\mathrm{d}}^{i}+t_{\text {frame }} \times W_{i}\right)
\end{aligned}
$$

where $t_{\mathrm{s}-\mathrm{d}}^{i}$ is the waiting time between reception and transmission slots on the $i$ th hop, e.g., if node 4 receives the packet from 1, it forwards the packet after $t_{\mathrm{s}-\mathrm{d}}=2 \times t$ slot $=30 \mathrm{~ms}$. Note that the first hop delay depends on the instants of packet generation and sending. Since they are uncorrelated, we use half a superframe length as the average waiting time. For shared slots MAC, instead of $t_{\mathrm{s}-\mathrm{d}}$, we use average back-off length in calculations. At the first hop, it might happen that the packet is sent in the same slot as it is generated, hence the -1 in Eqns. (4) and (3):

$$
\begin{array}{r}
d_{\min }=(n-1) \times t_{\text {slot }} \\
d_{\text {retx }}=\sum_{i=1}^{n} d_{\mathrm{hop}}^{i}=\sum_{i=1}^{n} \sum_{j=1}^{W_{i}} \frac{2^{j}}{2}-1,
\end{array}
$$

Using Eqns. (17)-(4), we can now split the delay into lossinduced, i.e., due to retransmissions only, and buffering time, i.e., waiting time in the buffer until other packets are served:

$$
\begin{aligned}
d_{\text {loss }} & =d_{\text {retx }}-d_{\text {min }} \\
d_{\text {buf }} & =d_{\text {p }}-d_{\text {retx }}
\end{aligned}
$$

These two delay parameters are coupled: if loss occurs, loss-induced delay, and, hence, average serving time of a packet, will increase, causing in turn longer buffering time. The split is exemplified in Fig. 7 for the scenario VII. While $d_{\min }$ is only path-dependent, $d_{\text {retx }}$ depends on the amount of

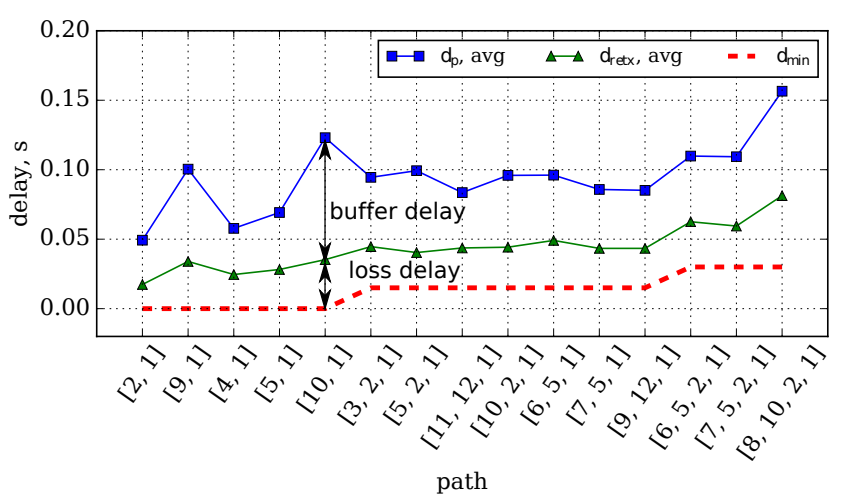

Fig. 7. Example of a path delay analysis from data set VII.

retransmissions on every hop for all packets on the path. The difference between them is, thus, $d_{\text {loss }}$, showing how large the delay for the given packet would be if it has not been waiting in the buffer for other transmissions. On the other hand, difference between the measured delay $d_{\mathrm{p}}$ and $d_{\text {retx }}$ shows how long has the packet been waiting in the buffer.

1) Path and hop delay: Fig. 8 represents overall delay for all scenarios. We observe that average delay is an order of magnitude higher for the reserved slots scenarios compared to shared slots, even for the high-load case. This results is expected: with the number of transmissions limited to 3 , the maximum link delay for a packet in contention-free access is $(13+4) \times 15=255 \mathrm{~ms}$, which is exactly three times less than the maximum serving time in the dedicated slots case (ignoring the buffer impact). Although induced interference measurements record 20\% larger load compared to no interference, the highest delay is always in the case of high-load scenario (IV and VIII). While these two trends are well expected, we also notice that the week-day measurements in II has $30 \%$ lower delay than weekend data set I, which contradicts to the expectation. Another observation is that for the high-load scenario, outliers have large impact on the 


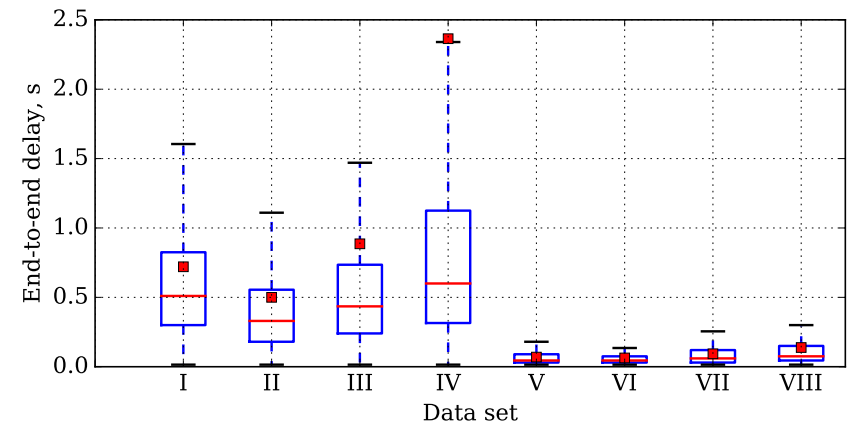

Fig. 8. Delay distribution for different scenarios (numeration according to Table II. For readability, no outliers are shown, however, they are considered while calculating mean values (red squares). Left four scenarios are for dedicated slots, right four - shared slots.

average delay, especially for contention-free scheme.

Next, we show the normalized delay $d_{\text {hop }}$ in Fig. 8. It is observed that, except for the high load scenarios, there is no significant difference in delay. However, in the high load scenarios, bursty applications experience up to 2.5 (contentionfree) and 1.2 (contention-based) higher delay.

2) Loss- and buffer-induced delay: Now, we apply the delay analysis to compare the normalized path delay for six data sets (leaving out II and VI for illustrative purposes), and the sets of paths which are common for all data sets (to exclude topology influence). Fig. 10 compares buffering and loss-induced delays for different scenarios. It is observed that the interference scenario influences both the delays, in approximately equal fashion. However, the high load scenario does not influence the loss-induced delay for both medium access schemes. This contradicts to the expectations: shared medium access is known to cause collisions, which in turn should increase the loss-induced delay. The effect can be explained by the fact that (1) medium utilization is still low, and, hence, amount of collisions is also low, and (2) the collision resolution is fast and effective, hence, it mitigated
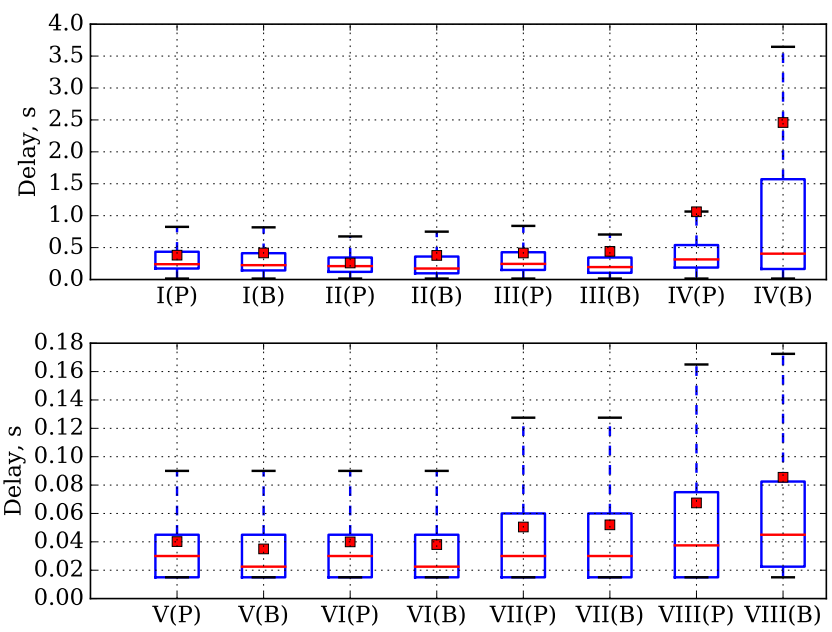

Fig. 9. Average hop delay distribution for periodic (labeled as P) and bursty (B) application: dedicated slots (above) and shared slots (below), see Table II
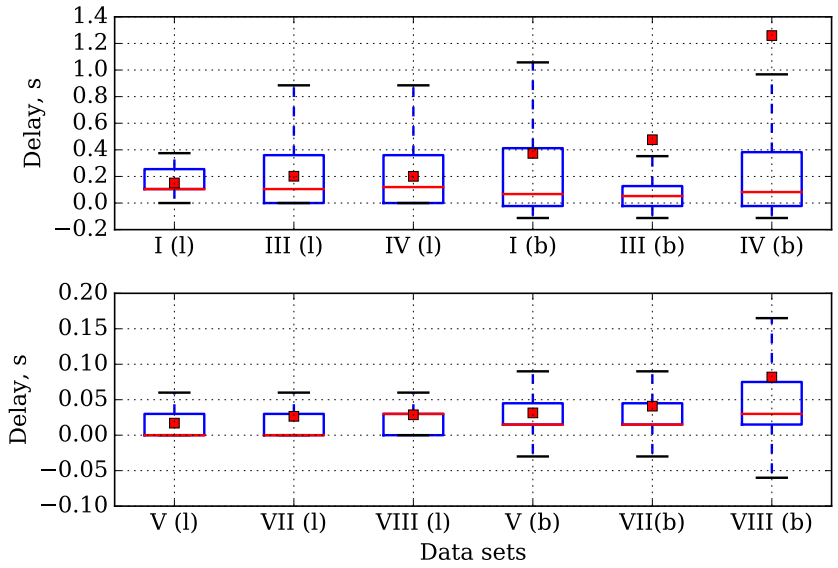

Fig. 10. The plot illustrates loss- and buffer-induced delay (subscripts 1 and b respectively). Note that since the first hop delay in calculated in average for $d_{\min }$ and $d_{\text {retx }}$, resulting buffering delay can be negative.

the impact on the delay. The last observation from the figure is that the buffering delay is influenced by the load in the network heavier than by the introduction of interference.

3) Delay and reliability correlation: As we show in the previous subsection, delay and reliability are interrelated via the retransmission. Fig. 11 serves to summarize this effect and show the correlation between them. The figure shows average delay for only most used paths selected (in order to filter out the paths with less samples). The delay is shown as an average per path, and the path reliability is calculated from the data in Fig. 6 as $R_{\text {path }}=\prod_{i=1}^{n} R_{i}$, where $R_{i}$ - packet success rate on the hop $i$. We can see similar correlation for both medium access schemes, but on a different scale: with the decrease in path reliability, average delay and the variation of the delay among different paths increase.

\section{Suitability FOR AMR}

Finally, in order to estimate how suitable 802.15.4e WSN is for automatic meter reading application, we have to know the
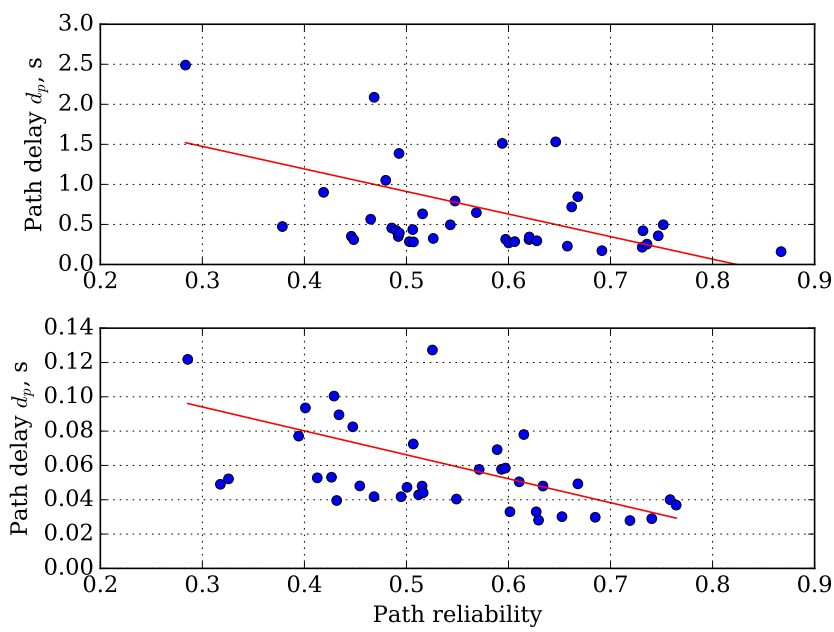

Fig. 11. Correlation between path delay and path reliability for dedicated slots (above), shared slots (below). 


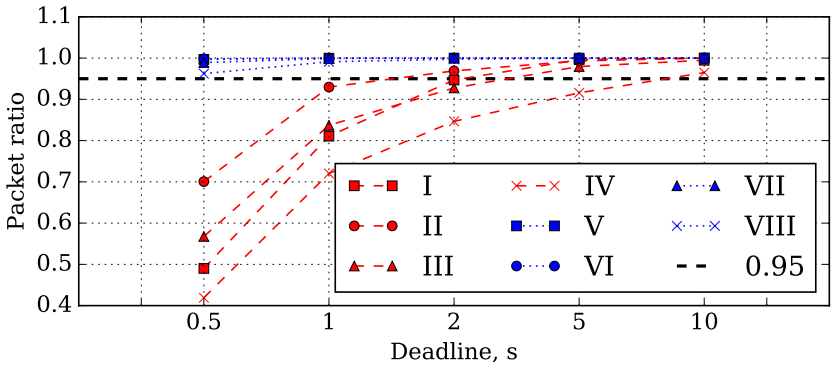

Fig. 12. Ratio of packets ( $\mathrm{y}$ axis) arriving within a given deadline ( $\mathrm{x}$ axis).

deadlines and delivery ratio requirements for AMR. There is unfortunately no consensus about this parameters in the literature. Common suggestions for PDR are 0.95 and 0.99, and the required deadline ranges from few hundred milliseconds [1] to several seconds [2]. In Fig. 12, we provide a comparison for packet ratio (y-axis) achieving given deadlines (x-axis) for all data sets. It is observed that 0.95 PDR is achieved already with $0.5 \mathrm{~s}$ deadline for the shared access, and only from $10 \mathrm{~s}$ for the dedicated slots access.

However, this plot does not take into account the reliability results from Subsec. V-B As we observe from Fig. 5, the required 0.95 PDR is not achieved in any scenario, although for certain motes in low load scenarios (I-III and V-VII), the requirement is satisfied. This points out that the reliability is the main drawback for WSN deployment in this scenario, and solely the frequency diversity provided by hopping is not sufficient. Additional means for ensuring high packet delivery ratio have to be developed. For example, it can be MAC layer retransmission, or transport protocols, such as TCP or SCTP. However, both of the methods involve a trade-off: they create additional load in the network, thus, also decreasing the reliability and increasing the delay. This trade-off has to be considered while designing the reliability mechanisms for metering data delivery.

\section{CONCLUSIONS}

In this paper, we have presented a testbed-based performance evaluation of $802.15 .4 \mathrm{e}$ for an exemplary AMR applications. We have conducted eight sets of measurements in a multi-hop network, for three interference scenarios (weekend, week day and high interference), two medium access variations of TSCH profile (with shared and with dedicated slots), and two load scenarios (high and low). The results show superiority of shared slots MAC compared to dedicated slots for our setup. Despite an increased collision ratio packet level reliability, end-to-end reliability has been shown to be similar for both cases, and the delay is an order of magnitude lower for shared slots. This suggests that, in order to keep delay low for TDMA-based medium access, more efficient, topology-aware means of defining the schedule should be designed.

We further evaluated the applicability of TSCH MAC for AMR applications. The evaluation points out, that the packet delivery ratio does not on average meet the required 0.95 , suggesting that better reliability mechanisms have to be deployed.
From the delay perspective, both MAC schemes can meet the deadline of $10 \mathrm{~s}$ with $95 \%$ assurance, while only shared slots MAC meets the $0.5 \mathrm{~s}$ requirement.

\section{REFERENCES}

[1] R. H. Khan and J. Y. Khan, "A comprehensive review of the application characteristics and traffic requirements of a smart grid communications network," Computer Networks, vol. 57, no. 3, pp. 825-845, 2013.

[2] V. C. Gungor, D. Sahin, T. Kocak, S. Ergut, C. Buccella, C. Cecati, and G. P. Hancke, "A survey on smart grid potential applications and communication requirements," IEEE Transactions on Industrial Informatics, vol. 9, no. 1, pp. 28-42, Feb 2013.

[3] T. Khalifa, K. Naik, and A. Nayak, "A survey of communication protocols for automatic meter reading applications," Communications Surveys \& Tutorials, IEEE, vol. 13, no. 2, pp. 168-182, 2011.

[4] J. Brown and J. Y. Khan, "Performance comparison of LTE FDD and TDD based smart grid communications networks for uplink biased traffic," in Smart Grid Communications, IEEE International Conference on. IEEE, 2012, pp. 276-281.

[5] U. Sulayman and A. T. Alouani, "Smart grid monitoring using local area sensor network. Real-time data acquisition, analysis and management," in 2011 Proceedings of IEEE Southeastcon, 2011.

[6] J. Markkula and J. Haapola, "LTE and hybrid sensor-LTE network performances in smart grid demand response scenarios," in Smart Grid Communications, 2013 IEEE International Conference on. IEEE, 2013, pp. 187-192.

[7] A. Osseiran, F. Boccardi, V. Braun, K. Kusume, P. Marsch, M. Maternia, O. Queseth, M. Schellmann, H. Schotten, H. Taoka et al., "Scenarios for $5 \mathrm{G}$ mobile and wireless communications: the vision of the METIS project," IEEE Communications Magazine, vol. 52, no. 5, pp. 26-35, 2014.

[8] M. Gürsu, M. Vilgelm, W. Kellerer, and E. Fazli, "A wireless technology assessment for reliable communication in aircraft," in Wireless for Space and Extreme Environments (WiSEE), 2015 IEEE International Conference on, Dec 2015, pp. 1-6.

[9] F. Chen, R. German, and F. Dressler, "Towards IEEE 802.15. 4e: a study of performance aspects," in Pervasive Computing and Communications Workshops (PERCOM Workshops), 2010 8th IEEE International Conference on. IEEE, 2010, pp. 68-73.

[10] G. Alderisi, G. Patti, O. Mirabella, and L. L. Bello, "Simulative assessments of the IEEE 802.15.4e DSME and TSCH in realistic process automation scenarios," in Industrial Informatics (INDIN), 2015 IEEE 13th International Conference on. IEEE, 2015, pp. 948-955.

[11] J. Blanckenstein, C. Nardin, J. Klaue, and H. Karl, "Error characterization of multi-access point WSNs in an aircraft cabin," in Communication Workshop (ICCW), 2015 IEEE International Conference on. IEEE, 2015, pp. 2363-2368.

[12] M. Gürsu, M. Vilgelm, S. Zoppi, and W. Kellerer, "Reliable co-existence of 802.15. 4e TSCH-based WSN and Wi-Fi in an Aircraft Cabin," in Communications, CROWD-Net Workshop, IEEE Int. Conf. on, 2016.

[13] A. Gonga, O. Landsiedel, P. Soldati, and M. Johansson, "Revisiting multi-channel communication to mitigate interference and link dynamics in wireless sensor networks," in Distributed Computing in Sensor Systems (DCOSS), 2012 IEEE 8th International Conference on. IEEE, 2012, pp. 186-193.

[14] T. Winter, "Routing Protocol for Low-Power and Lossy Networks," RFC 6550, 6551, 6552. IETF, Tech. Rep., 2012.

[15] J. Vasseur et al., "Routing metrics used for path calculation in low-power and lossy networks," RFC6551, 2012.

[16] R. H. Khan, K. Mahata, and J. Brown, "An adaptive RRM scheme for smart grid M2M applications over a WiMAX network," in Communication Systems, Networks \& Digital Signal Processing (CSNDSP), 2014 9th International Symposium on. IEEE, 2014, pp. 820-825.

[17] T. Watteyne, X. Vilajosana, B. Kerkez, F. Chraim, K. Weekly, Q. Wang, S. Glaser, and K. Pister, "OpenWSN: a standards-based low-power wireless development environment," Transactions on Emerging Telecommunications Technologies, vol. 23, no. 5, pp. 480-493, 2012. 